\title{
Comparing the delivery to the hair bulb of two fluorescent molecules of distinct hydrophilicities by different nanoparticles and a serum formulation
}

\author{
Cristiana Costa ${ }^{\text {a }}$, Bruno Fernandes ${ }^{\text {a }}$, Diana Guimarães ${ }^{\text {a }}$, Eugénia Nogueira ${ }^{\text {a,b }}$, \\ Madalena Martins ${ }^{\mathrm{a}, \mathrm{b}}$, Teresa Matamá ${ }^{\mathrm{a}, *}$, Artur Cavaco-Paulo ${ }^{\mathrm{a},}$ \\ ${ }^{a}$ CEB - Centre of Biological Engineering, University of Minho, 4710-057 Braga, Portugal \\ ${ }^{\mathrm{b}}$ Solfarcos - Pharmaceutical and Cosmetic Solutions Ltd, Praceta do Vilar, Urbanização Quinta dos Órfãos, Bloco A - Loja 6, 4710-453 Braga, Portugal
}

\section{A R T I C L E I N F O}

\section{Keywords:}

Topical delivery

Follicular penetration

Nanoparticle systems

Formulations

Hair follicles

Hair bulb

\begin{abstract}
A B S T R A C T
The follicular route is an important drug penetration pathway in any topical application, either concerning dermatological and cosmetic skin treatments or any transdermal administration regimen. Efficient transport into follicles will depend on drug inherent properties but also on the chosen vehicle. The main study goal was to compare several systems for the delivery to the hair bulb of two fluorescent molecules of different water affinities: the hydrophobic Nile Red and the quite similar but hydrophilic Nile Blue. Three common nanoparticle types were compared in terms of encapsulation efficiency and stability: liposomes, ethosomes and polymeric nanoparticles. A liquid serum-like formulation was also developed, adjusting the final ethanol amount to the type of dye to be solubilized. Then, this formulation and the nanoparticle systems that successfully passed characterization and stability stages were further studied on their ability to reach the bulb. The serum formulation was able to deliver, both drug models, to deeper follicular regions than nanoparticles. Attending to the envisioned zone target of the follicle, the simplest approach proved to be the best choice from all the systems tested in this work. Nonetheless, nanocarriers and the inherent complexity of their manufacturing processes may be justified under very specific requirements.
\end{abstract}

\section{Introduction}

Improved bioavailability of topically applied drugs has been a major pharmaceutical aim. Though skin seems an ideal therapeutic target organ by being easily accessible, usually not associated with increased plasma levels, gastrointestinal/enzymatic drug degradation, hepatic first pass metabolism neither with systemic toxicity and side effects, it is an amazing barrier (Patzelt and Lademann, 2020). Skin prevents the penetration of most topically applied drugs resulting in a general low bioavailability.

In skin, hair follicles (HFs) are an important and promising penetration pathway for dermatotherapy and they have been intensively investigated for more than 20 years (Lademann et al., 2008). HFs can be an efficient drug reservoir, creating the possibility for sustained drug delivery (Schafer-Korting et al., 2007). Indeed, the compounds and particles have a significantly longer storage time when they are inside
HFs than when they stay trapped in the stratum corneum (Lademann et al., 2006). Besides their importance in topical drug administration, HFs can their selves be a treatment target: bulb, bulge or associated sebaceous glands. Follicular permeation and accumulation of topically applied substances is particularly critical for the efficacy of skin medical and cosmetic treatments (Ossadnik et al., 2006). The HFs occupy $<0.1 \%$ of total skin surface area, however, on scalp and face regions, they can be as much as $10 \%$ of total skin area (Fang et al., 2014; Wosicka and Cal, 2010). The region of the infundibulum on HFs increases the body surface area and interrupts the epidermal barrier towards the lower parts of the $\mathrm{HF}$, serving as an entry access for drug delivery (Fang et al., 2014).

Efficient drug transport into the HFs depends upon the interaction between the drug and the sebum as well as the choice of the drug vehicle (Blume-Peytavi and Vogt, 2011). Nanotechnology is a modern and quickly evolving trend in dermal, transdermal and follicular drug delivery; there are a huge variety of nanocarriers, including liposomes,

Abbreviations: DOPE, 1,2-dioleoyl-sn-glycero-3-phosphoethanolamine; DSPE-mPEG, 1,2-distearoyl-sn-glycero-3-phosphoethanolamine-N-[amino(polyethylene

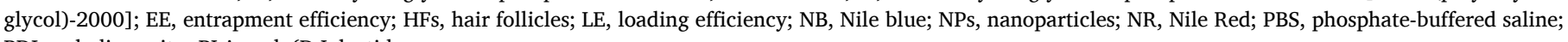
PDI, polydispersity; PLA, poly(D,L-lactide.

* Corresponding authors.

E-mail addresses: teresam@ceb.uminho.pt (T. Matamá), artur@deb.uminho.pt (A. Cavaco-Paulo). 
nano-formulations, nanocrystals, polymeric nanoparticles, among others (Czajkowska-Kośnik et al., 2019). General advantages of nanoparticle-based topical delivery systems are the transport of increased drug concentrations, better solubility of hydrophobic drugs, increased chemical and physical stability and both sustained and controlled release of the cargo (Patzelt and Lademann, 2020). In fact, nanoparticles can improve the stability of several molecules (Morganti, 2010; Rosen et al., 2015), promote their accumulation in the follicular opening and enhance their permeation along the follicular duct. Besides clinical applications, drug encapsulation systems are also key tools on the development of new cosmetic products. Cosmetic products are chemically complex formulations where the long-term stability of ingredients is a major issue.

There are several reported studies that analyze the skin and follicular permeation of drug delivery systems (Abd et al., 2018; Blume-Peytavi et al., 2010; Lauterbach and Müller-Goymann, 2015; Ossadnik et al., 2006; Patzelt et al., 2011; Teichmann et al., 2007). Recently, some nanocarriers were developed to deliver topically drugs with interesting potential to treat acne vulgaris, hidradenitis suppurativa, androgenic alopecia and inflammation-based skin and hair disorders (Angelo et al., 2020; Pereira et al., 2021; Tolentino et al., 2021; Ushirobira et al., 2020). The authors have compared the developed vehicles with commercial ones or with the drug in solution and they have elegantly quantified which percentage of the drug penetrated into the skin specifically through the follicular pathway (Angelo et al., 2020; Pereira et al., 2021; Tolentino et al., 2021; Ushirobira et al., 2020). The different nanoparticles and nanocapsules all promoted the targeting of the HFs regarding the other non-structured vehicles. Most of the research done in the area of topical drug administration, using either nanocarriers or vehicle formulations, is designed to deliver the cargo into the systemic circulation, to skin layers using the follicular route (thus avoiding the stratum corneum barrier) or to particular follicle regions not as deep as the bulb. With this work we aimed to deliver molecules with different water affinities to the bulb region of hair follicles. Hair bulbs contain the cell populations responsible for the production, morphology and pigmentation of the hair fiber and therefore are important cosmetic targets (Knorr et al., 2009; Wosicka and Cal, 2010). Our approach consisted in comparing the performance of different but common vehicles for structurally very similar cargos: two fluorescent molecules, one being used as a model for hydrophilic and the other as a model for hydrophobic compounds. Three different drug encapsulation systems liposomes, ethosomes, and polymeric nanoparticles - and a new developed formulation were compared. The formulation was developed as a scalp serum with common cosmetic ingredients in which the solubility of the two fluorescent dyes was attained with different amounts of ethanol. First, we characterized the physical properties of each particulate system and their stability over time. The particles that were most suited to encapsulate the model molecules and that remained stable over several months of storage were further studied in terms of hair follicle penetration and compared to the serum-like formulation. We studied qualitatively the skin and HF penetration profile of the model molecules using ex vivo porcine skin, still considered one of the best human skin models (Lauterbach and Müller-Goymann, 2015).

\section{Materials and methods}

\subsection{Chemicals and solvents}

The materials Poly (D,L-lactide) (PLA) ( $\mathrm{Mw}=18-24 \mathrm{kDa}$ ), Pluronic ${ }^{\circledR}$ F68 (suitable for cell culture), Nile Blue (NB, Basic Blue 12) (Mw $=732.8 \mathrm{~g} / \mathrm{mol}$; predicted $\log \mathrm{P}=0.7$ according to Molinspiration Cheminformatics free web services, https://www.molinspiration.com, Slovensky Grob, Slovakia) and cholesterol were purchased from SigmaAldrich (Sigma Chemical Co., St. Louis, MO, U.S.A.). Nile Red (NR, Nile Blue oxazone) ( $\mathrm{Mw}=318.4 \mathrm{~g} / \mathrm{mol}$; predicted $\log \mathrm{P}=4.58$ according to Molinspiration Cheminformatics free web services, https://www. molinspiration.com, Slovensky Grob, Slovakia) was purchased from TCI (Tokyo Chemical Industry Co., Ltd., Tokyo, Japan). 2-Propanol was purchased from Fisher Scientific (Hampton, NH, U.S.A). 1,2-dioleoyl-snglycero-3-phosphoethanolamine (DOPE), and 1,2-distearoyl-sn-glycero3-phosphoethanolamine- $\mathrm{N}$-[amino(polyethylene glycol)-2000] (DSPEmPEG) were purchased from Lipoid GmbH (Ludwigshafen am Rhein, Germany). Acetone and ethanol were of the highest grade commercially available and purchased from several suppliers.

\subsection{Preparation of the delivery systems}

Three drug encapsulation systems were prepared: two lipid-based nanocarriers and PLA nanoparticles (PLA NPs). By mixing four common commercial cosmetic ingredients, a non-particulate serum, profiled as a scalp serum, was obtained in collaboration with subcontractor company - Inovapotek, Pharmaceutical Research and Development, Lda. (Porto, Portugal).

PLA NPs were prepared using the nanoprecipitation protocol described by Fernandes et al. (Fernandes et al., 2015). Firstly, the polymer was dissolved in a mixture of ethanol and acetone $(50: 50, \mathrm{v} / \mathrm{v})$ at a concentration of $2 \%(\mathrm{w} / \mathrm{w})$. Then, under magnetic stirring, one volume of the organic phase was added to two volumes of an aqueous phase containing $0.6 \%(\mathrm{w} / \mathrm{w})$ Pluronic ${ }^{\circledR}$ F68. After nanoparticles have formed, the organic phase was completely removed with a rotary evaporator. The aqueous suspensions of nanoparticles, containing approximately $0.8 \%$ PLA, (w/w) were stored at $4{ }^{\circ} \mathrm{C}$. PLA NPs loaded with the fluorescent dyes (final concentration of $100 \mu \mathrm{g} / \mathrm{ml}$ ) were obtained as above by dissolving NR in the organic phase or NB in the aqueous phase. Care was taken to avoid exposure to light throughout all the experimental procedures involving the fluorescent dyes.

Liposomes were prepared with the modified ethanol injection method described by Guimarães et al. (Guimarães et al., 2020), using DOPE, cholesterol and DSPE-mPEG at a molar ratio of $5.4: 3.6: 1$, respectively. Briefly, these components were first dissolved in ethanol and one volume of the ethanolic solution was injected under agitation (500 rpm) into one volume of the aqueous phase [phosphate-buffered saline (PBS), $\mathrm{pH} 7.4,70^{\circ} \mathrm{C}$ ]. The encapsulation of dyes was attained by solubilizing them in the organic or aqueous phase, as described for the preparation of PLA NPs. The liposomal formulations were kept under agitation, at $70{ }^{\circ} \mathrm{C}$, to evaporate the organic phase. Afterwards, PBS was added to achieve a final concentration of $1.31 \%(w / w)$ DOPE, $0.45 \%$ $(\mathrm{w} / \mathrm{w})$ cholesterol and $0.91 \%(\mathrm{w} / \mathrm{w})$ DSPE-mPEG. The prepared liposomal formulations were stored at $4{ }^{\circ} \mathrm{C}$.

Ethosomes were prepared using the same methodology as described for liposomes, but, when adjusting the final concentration of particles with PBS, ethanol was added to a final concentration of $10 \%(\mathrm{v} / \mathrm{v})$.

To remove the free dye in all particulate systems, the suspensions were passed through a gel filtration chromatography column with a 5 kDa cut-off (PD-10 Desalting Columns containing $8.3 \mathrm{~mL}$ of Sephadex ${ }^{\mathrm{TM}}$ G-25 Medium; GE Healthcare, IL, U.S.A.). The separation was performed according to the supplier's instructions. The columns were equilibrated with water, PBS or $10 \%(\mathrm{v} / \mathrm{v})$ ethanol in PBS, according to the type of particles, i.e., PLA NPs, liposomes or ethosomes; the collected particle suspensions were 1.4 times more diluted than before gel filtration.

The serum-like formulation - consisted of an aqueous mixture of denatured ethanol, a preservative, a permeating and a thickener. Two formulations were developed taking into account the fluorescent molecule needed to be stably solubilized in it. Both contained $0.1 \%(\mathrm{v} / \mathrm{v})$ Lecigel $^{\mathrm{TM}}$ (used as emulsifier, penetration enhancer and gelling agent), $1 \%(\mathrm{v} / \mathrm{v})$ Euxyl ${ }^{\circledR}$ PE9010 (used as preservative) and 4\% (v/v) Transcutol ${ }^{\circledR}$ CG (used as penetration enhancer). In the formulation containing $40 \mu \mathrm{g} / \mathrm{mL} \mathrm{NB}$, the final concentration of ethanol was set at $10 \%(\mathrm{v} / \mathrm{v})$. In the formulation containing $40 \mu \mathrm{g} / \mathrm{mL} \mathrm{NR}$, the final concentration of ethanol was set at $53 \%(\mathrm{v} / \mathrm{v})$. 


\subsection{Particle size and size distribution}

The mean size (nm) and polydispersity (PDI, index from 0.0 to 1.0 ) of purified PLA NPs, liposomes and ethosomes were assessed by Photon Correlation Spectroscopy (PCS). All measurements were performed at $25 \pm 1{ }^{\circ} \mathrm{C}$, using a ZetaSizer Nano ZS equipment (Malvern Instruments Ltd, Worcestershire, U.K.). The physical stability of suspensions was evaluated over 6 months, by measuring the size and PDI of all nanoparticles stored at $4{ }^{\circ} \mathrm{C}$.

\subsection{Entrapment efficiency determination}

The liposomes and ethosomes were lysed with isopropanol. The PLA NPs were lysed with acetone. The encapsulated dye in all particulate systems was assessed by Ultraviolet (UV)-Spectrophotometry. For NRloaded PLA NPs, measurements were performed with an excitation wavelength at $536 \mathrm{~nm}$ and emission wavelength at $608 \mathrm{~nm}$. For NBloaded PLA NPs, $499 \mathrm{~nm}$ and $596 \mathrm{~nm}$ were used as excitation and emission wavelengths, respectively. For NR-loaded liposomes or ethosomes, measurements were performed with an excitation wavelength at $552 \mathrm{~nm}$ and emission wavelength at $635 \mathrm{~nm}$. For NB-loaded liposomes or ethosomes, $635 \mathrm{~nm}$ and $680 \mathrm{~nm}$ were used as excitation and emission wavelengths, respectively. The amount of dyes was determined using calibration curves. The percentages of entrapment efficiency (EE \%) were calculated with respect to the initial dye amount present in the non-purified suspensions of nanoparticles, according to the following equation (1):

$E E \%=\frac{[\text { mass of dye in nanoparticles }]}{[\text { initial mass of dye }]} \times 100$

The entrapment efficiencies were reevaluated periodically over 6 months storage at $4{ }^{\circ} \mathrm{C}$, to infer about the chemical stability of compound-loaded nanoparticles suspensions. Before each evaluation, the suspensions were filtrated again, as described before, to remove free dyes.

The percentages of loading efficiency (LE \%) were also calculated with respect to the amount of lipids or polymer used in the preparation of nanoparticles, according to the following equation (2):

$\mathrm{LE} \%=\frac{[\text { mass of dye in nanoparticles }]}{[\text { mass of nanoparticles }]} \times 100$

\subsection{Ex vivo HF penetration and distribution assay}

The porcine skin samples were excised from the abdominal region of freshly slaughtered pigs. Approval for these experiments was obtained from the Portuguese competent authority, the Direção Geral de Veterinária. Pre-treatment of porcine skin included a rinse under running tap water, a careful removal of subcutaneous fat, and a final rinse with sterile PBS ( $\mathrm{pH}$ 7.4). Skin pieces were placed with the stratum corneum facing the donor compartment and the dermis facing the receptor compartment of Franz Diffusion Cells with a diffusional area of $0.64 \mathrm{~cm}^{2}$ (PermeGear Inc., Bethlehem, PA, U.S.A.). After tissue acclimatization to the receptor phase (PBS, $\mathrm{pH} 7.4$, thermostatically maintained at $37^{\circ} \mathrm{C}$ ),
$300 \mu \mathrm{L}$ of each dye-loaded NPs suspension or serum-like formulation were applied, in a single dose, to the skin surface. The experiments were conducted for $24 \mathrm{~h}$ with at least 2 replicates. After $24 \mathrm{~h}$, the skin samples were rapidly washed and quickly frozen in optimal cutting temperature compound (OCT medium, Thermo Fisher Scientific, Waltham, MA, U.S. A.) and kept at $-80{ }^{\circ} \mathrm{C}$. Histological cuts of $20 \mu \mathrm{m}$ thickness were made using a Leica CM1900 cryostat (Leica Microsystems, Numsloch, Germany). Each sample resulted in, at least, 50 histological sections. The penetration and distribution of the fluorescent dyes were investigated in skin sections by brightfield and fluorescence microscopy (Olympus BX51 Microscope; Olympus Corporation, Tokyo, Japan). The exposure times used were defined respecting the maximal exposure times of histological sections from control samples at which there was a complete absence of skin auto fluorescence. Control samples were ex vivo porcine skin pieces treated with empty NPs systems and scalp serum formulation, under the same conditions as previously described.

\subsection{Statistical analysis}

All the results are expressed as mean value \pm standard deviation (SD) of at least three experiments. GraphPad Prism, version 8 (GraphPad Software, CA, U.S.A.) was used to perform the statistical analysis of the experimental data, using analysis of variance (ANOVA) and unpaired Student $t$-test. The differences were considered significant at $\mathrm{p}$-values $\leq$ 0.05 .

\section{Results and discussion}

In view of the topical delivery of hydrophobic and hydrophilic bioactive molecules to the hair bulb, the work started with the preparation and characterization of several particulate vehicle systems. For the selection of the best systems, the considered criteria were their ability to encapsulate the chosen model molecules, the stability of that encapsulation as well as the physical properties of the particles. Achieving the bulb of the HFs is of major interest for us, since it is the region where hair fiber synthesis and its natural phenotype setting occur.

\subsection{Preparation and characterization of the particulate delivery systems}

Table 1 summarizes the average size and size distribution of the prepared empty and dye-loaded NP systems. The particle Z-average values for both lipid-based systems are very similar, while for PLA NPs they are slightly higher; all values are in the range of sizes obtained before (Fernandes et al., 2015; Guimarães et al., 2020). A tendency for particle shrinkage or enlargement is also observed in all tested systems when loaded with NR or NB, respectively; the only exception concerns the NB-loaded ethosomes, that maintained their size. Regarding the polydispersity, the values obtained are very low and all the produced systems can be considered monodispersed. Nonetheless, the PLA NPs present the greatest size homogeneity. Although significant differences were detected statistically, in practical terms, they would not noticeably interfere with the ability of nanoparticles to penetrate into and along the follicle.

Table 1

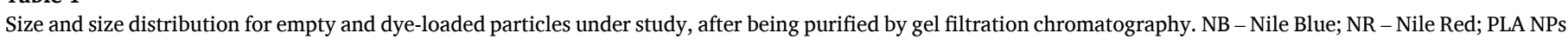

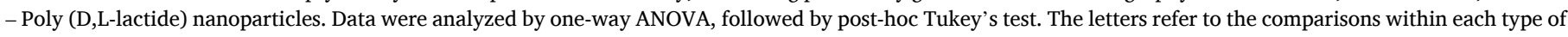
particles. The symbols refer to the comparisons between particles. Means that do not share a letter or a symbol are significantly different, $\mathrm{p} \leq 0.05$.

\begin{tabular}{|c|c|c|c|c|}
\hline & Dye & Liposomes & Ethosomes & PLA NPs \\
\hline \multirow[t]{3}{*}{ Z-average \pm SD $(\mathrm{nm})$} & - & $128 \pm 2^{\mathrm{a}^{*}}$ & $131 \pm 4^{\mathrm{a}^{*}}$ & $165.1 \pm 0.6^{\mathrm{a}+}$ \\
\hline & NR & $121.8 \pm 0.4^{\mathrm{b}^{*}}$ & $124 \pm 6^{\mathrm{a}^{*}}$ & $148 \pm 1^{\mathrm{b}+}$ \\
\hline & NB & $138 \pm 3^{\mathrm{c}^{*}}$ & $125 \pm 2^{a+}$ & $176 \pm 2^{c \varnothing}$ \\
\hline \multirow[t]{3}{*}{$\mathrm{PDI} \pm \mathrm{SD}$} & - & $0.118 \pm 0.001^{\mathrm{a}^{*}}$ & $0.108 \pm 0.005^{a+}$ & $0.095 \pm 0.004^{\mathrm{a} \varnothing}$ \\
\hline & NR & $0.114 \pm 0.004^{\mathrm{ab}^{*}}$ & $0.103 \pm 0.008^{\mathrm{a}^{*}+}$ & $0.086 \pm 0.008^{a+}$ \\
\hline & NB & $0.107 \pm 0.005^{b^{*}}$ & $0.131 \pm 0.007^{\mathrm{b}+}$ & $0.084 \pm 0.003^{a \varnothing \varnothing}$ \\
\hline
\end{tabular}


Liposomes are vesicular structures with an aqueous core surrounded by a lipid bilayer membrane of non-toxic and biodegradable phospholipids (Bozzuto and Molinari, 2015; Ghanbarzadeh and Arami, 2013). They are widely studied for their ability to encapsulate both hydrophilic and lipophilic drugs, becoming a potential vehicle to deliver bioactive molecules into specific target sites with very promising results. In the particular case of HFs, the phospholipids of liposomes can assist the interaction with sebum and subsequently facilitate the entrance and delivery to this skin appendage (Jung et al., 2006; Kumar and Banga, 2016; Santos et al., 2018).

Ethosomes can have some advantages over liposomes in topical delivery. The ethanol per se has the ability to enhance the permeability of several compounds (both hydrophobic and hydrophilic) after topical application and improve their follicular penetration. Ethanol can interact with sebum and increase its fluidity just enough to enable a more effective penetration of the applied substances (Pilch and Musiał, 2018; Sala et al., 2018). Ethanol can also increase the flexibility and softness of liposomes and decrease their sizes (Fang et al., 2014; Ghanbarzadeh and Arami, 2013). In the present work, the concentration of ethanol used to produce the ethosomes did not affect the size of the particles with the chosen composition described in the methods section. The amount of ethanol applied to the liposomes was used in order to improve their follicular penetration.

Nanoparticles of PLA were also studied, already knowing their high potential for delivering compounds deeply into HFs (Fernandes et al., 2015). PLA is characterized as a linear, lipophilic, biodegradable polymer. The constituting monomer, D,L-lactic acid, can be easily obtained from renewable sources as the sugarcane or corn starch. Lactic acid is the primary degradation product of the complete polymer hydrolysis, which makes PLA of great interest for numerous applications (Rancan et al., 2009). The prepared PLA NPs presented a slightly higher size compared to the conventional liposomes and ethosomes; this size range obtained for PLA NPs is in accordance with Fernandes et al., these nanoparticles were capable of reaching the HFs bulbs (Fernandes et al., 2015).

Several reports in the literature state that it is possible to do a selective targeting of a particular structure or region within the $\mathrm{HF}$ by tuning the particle diameter (Rancan et al., 2009; Vogt et al., 2005; Wosicka and Cal, 2010). Indeed, it was already demonstrated by some authors, that the penetration depth increases within the follicular duct with decreasing size of the nanoparticles (Papakostas et al., 2011; Vogt et al., 2006). In addition, if nanoparticles are larger than $100 \mathrm{~nm}$ they will not translocate to other viable skin layers and will be confined inside the follicle duct (Rancan et al., 2009). According to the average sizes here obtained, all the prepared particles are below $200 \mathrm{~nm}$ which is an ideal size according to the work purpose. Regarding size distribution, all of the prepared NPs showed an even distribution with high homogeneity $(<0,140)$. According to Danaei et al., values of PDI $<0.2$ are usually considered acceptable for polymeric NPs (Danaei et al., 2018). For lipid-based nanocarriers, a PDI $<0.3$ is considered to be tolerable and designates a homogenous population (Badran, 2014; Chen et al., 2011; Putri et al., 2017). All the systems produced in this work possess adequate particle size and size distributions for topical follicular delivery of the model compounds, targeting the bulb.

\subsection{Entrapment efficiency of model compounds}

Two fluorescent dyes with very similar structures but with distinct water affinities were chosen in order to better evaluate the influence of the hydro- and lipophilic characters of the cargo on the entrapment efficiency and stability of NPs. Being fluorescent just serves the purpose of histological detection to study cargo penetration and distribution into the HF structure after topical application. In this sense, NB and NR were used as models of lipophilic and hydrophilic compounds, respectively. These dyes have good photostability and they can be incorporated into diverse materials or onto surfaces where they act as a light/pH sensors for various applications (Martinez and Henary, 2016). The NR molecule is intensely fluorescent in apolar media. However, in water, it is extremely poorly soluble (hydrophobic feature), and it does not show any fluorescence. NB is a cationic fluorescent molecule and thus, more readily soluble in water than NR (hydrophilic feature) (Martinez and Henary, 2016). Moreover, NR has its emission in the red region of the light spectrum, the region where skin shows low auto-fluorescence (Rancan et al., 2009), and NB, compared to NR, has a redshift in the absorbance spectrum making it even a better candidate for biological imaging (Martinez and Henary, 2016).

The results of dye encapsulation by the different lipid-based approaches are shown in Table 2. The encapsulation of both dyes into liposomes and ethosomes proved to be highly efficient. Around $100 \%$ of each dye was entrapped into these lipid nanoparticles, meaning that the two types of lipid-based particles are able, in theory, to transport both dyes. Besides, their loading efficiencies are also similar.

In this work, the entrapment efficiencies of the dyes by PLA NPs were around $30 \%$ for NB and around $10 \%$ for NR, considerably lower values compared to those obtained with lipid-based nanoparticles (Table 2). Such low EE can be related to the saturation of the system. If the initial amount of dyes used to prepare the dye-loaded PLA NPs was adjusted to near or below what seems to be the maximal entrapment capacity of those particles (Fernandes et al., 2015), the EE values would certainly be improved. Despite the low encapsulation efficiency, the theoretical loading efficiency of NB is very similar to the lipidic systems: $\approx 0.38 \mathrm{mg}$ of encapsulated dye per $100 \mathrm{mg}$ of polymer used in the preparation of nanoparticles. Furthermore, the NR-loaded PLA NPs prepared in this work, had a loading efficiency higher than the one previously obtained for these nanoparticles with this specific fluorescent molecule (Fernandes et al., 2015).

\subsection{Stability of particulate delivery systems}

The dye content of all prepared NPs was evaluated over 6 months of storage at $4{ }^{\circ} \mathrm{C}$ (Fig. 1).

For the lipid-based particles, the EE stability depends on the entrapped dye; PLA NPs were resilient to the hydro- and lipophilicity character of the dye (Fig. 1). The liposomes prepared with NR lost more than $60 \%$ of the dye content after 3 months; the lost amount did not significantly change after three more months (6 months in total). Although they kept the stability parameters of size and PDI (Table A.1) between acceptable levels, they were not able to retain the initial

Table 2

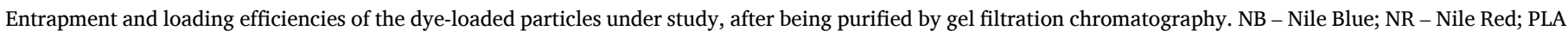

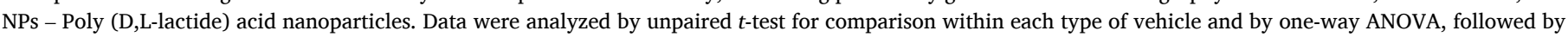

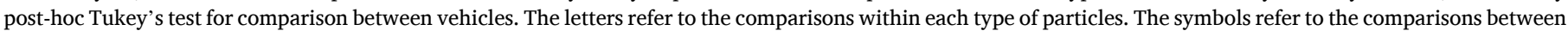
particles. Means that do not share a letter or a symbol are significantly different, $\mathrm{p} \leq 0.05$.

\begin{tabular}{|c|c|c|c|c|}
\hline & Dye & Liposomes & Ethosomes & PLA NPs \\
\hline \multirow[t]{2}{*}{ Entrapment Efficiency (\%) } & NR & $107 \pm 5^{\mathrm{a}^{*}}$ & $95.9 \pm 0.7^{\mathrm{a}^{*}}$ & $11.07 \pm 0.01^{\mathrm{a}+}$ \\
\hline & NB & $100 \pm 3^{\mathrm{a}^{*}}$ & $95 \pm 10^{\mathrm{a}^{*}}$ & $30.55 \pm 0.03^{b+}$ \\
\hline \multirow[t]{2}{*}{ Loading Efficiency (\%) } & NR & $0.39 \pm 0.02^{\mathrm{a}^{*}}$ & $0.350 \pm 0.003^{a^{*}}$ & $0.1384 \pm 0.0001^{\mathrm{a}+}$ \\
\hline & NB & $0.37 \pm 0.01^{\mathrm{a}^{*}}$ & $0.35 \pm 0.04^{\mathrm{a}^{*}}$ & $0.3819 \pm 0.0004^{b^{*}}$ \\
\hline
\end{tabular}



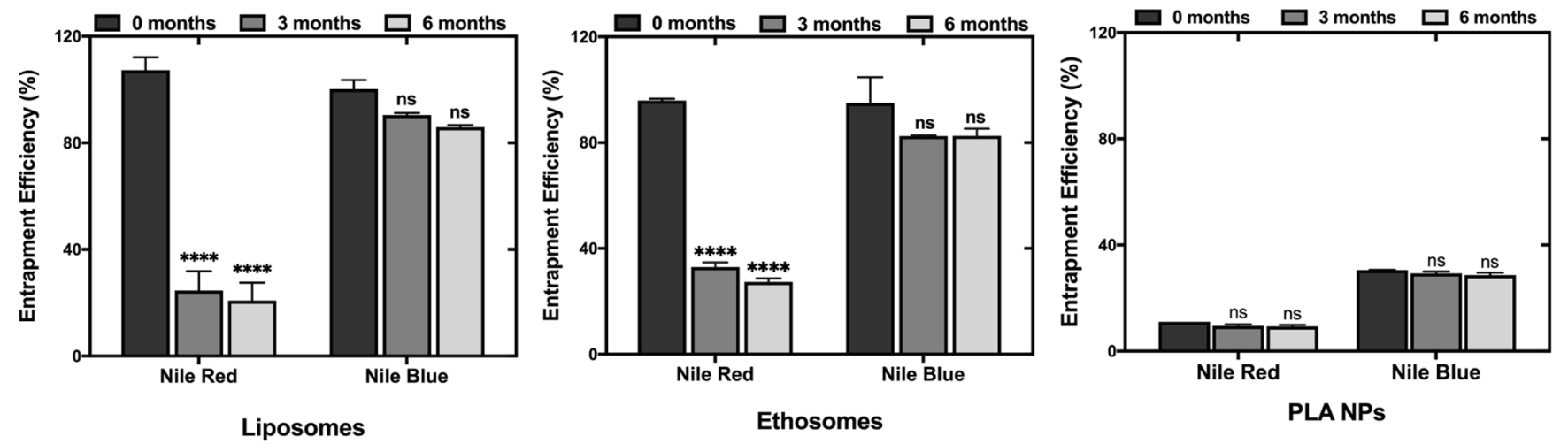

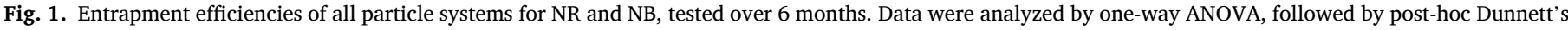
test. $* * * * \mathrm{p} \leq 0.0001$, compared with the time point 0 months; $\mathrm{ns}=$ not significant.

amount of dye. Nonetheless, it is important to emphasize that encapsulation and its stability depend on the physicochemical properties of both cargo and vehicle compounds, whatever the particulate system. Ethosomes loaded with NR presented the same issues detected for liposomes. They lost most of the initial dye amount, maintaining only about $30 \%$ of the dye after 6 months. Despite this, as for NR-loaded liposomes, NR-loaded ethosomes were also able to maintain their size and PDI parameters over the 6 months, with non-significant differences (Table A1).

In contrast to NR, a higher NB content was retained in liposomes; after a storage period of 6 months, liposomes contained more than $80 \%$ of the initial NB amount and they were also more stable in terms of size and size distribution (Table A1). Similar results were observed for NB- loaded ethosomes, where only a low percentage of dye was also lost and an increase in particles size $(\approx 35 \mathrm{~nm})$ was verified at the end of the storage period. In this way, both lipid-based systems loaded with the hydrophilic NB were chosen for subsequent ex vivo permeation studies.

Further optimizations for the NR-loaded liposomes and ethosomes must be performed in order to increase their physical stability. However, the investment of time and effort needed was out of the scope of this work. These optimizations could, for example, involve the adjustment of the initial NR concentration as well as optimizing the proportion of lipid constituents.

Although the PLA NPs presented low NR and NB entrapment efficiencies, they were able to stably retain the initial amount of encapsulated dyes throughout the 6 months (Fig. 1). Dye-loaded PLA NPs

A
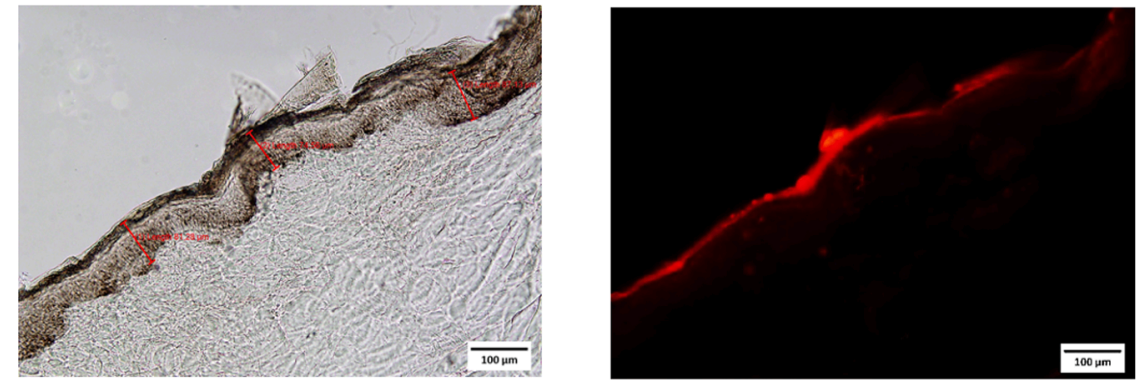

B

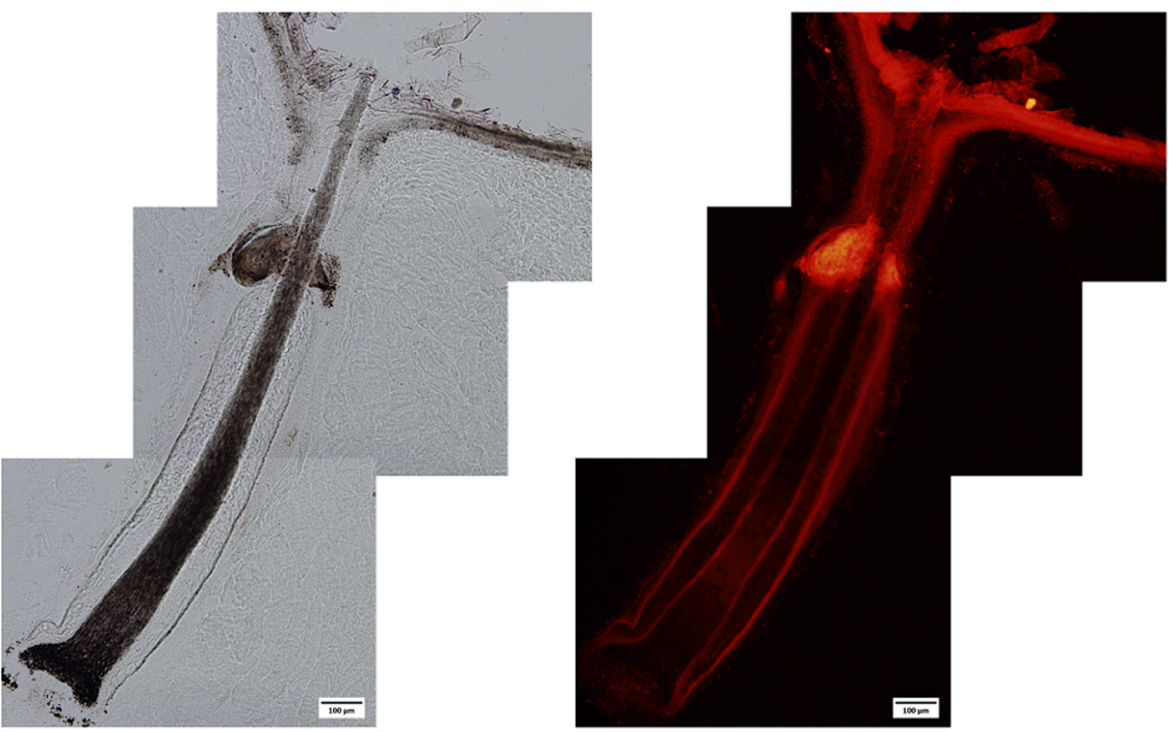

Fig. 2. Brightfield (left) and fluorescence (right) images of stratum corneum (A) and hair follicles (B) of cross-sectioned porcine skin, after $24 \mathrm{~h}$ of treatment with a scalp serum formulation containing the fluorescent molecule Nile Red (Bar $=100 \mu \mathrm{m}, 30 \mathrm{~ms}$ of exposure). 
slightly change its size keeping its PDI stable (Table A1). But, due to the low amount of the dyes encapsulated, the PLA NPs were not further studied.

The dye-encapsulation NP systems that presented the desired minimum stability were selected for further experiments. They were applied topically to ex vivo porcine skin and qualitatively compared with the serum-like formulation in terms of skin permeation profile.

\subsection{Penetration and distribution of model compounds in ex vivo porcine} skin

The skin distribution and follicular penetration depth of the topically applied fluorescent dyes, carried by drug encapsulation NPs systems and formulation, were assessed on ex vivo porcine skin (Figs. 2-5). Porcine skin has been considered a good and suitable skin model and has been extensively used to study the skin permeation since it resembles human skin regarding its biochemical composition, thickness, as well as, its permeability properties (Hammond et al., 2000; Lauterbach and MüillerGoymann, 2015; Mittal et al., 2013; Simon and Maibach, 2000). For the analysis of follicular penetration and dye distribution in porcine skin cryo-sections, the exposure times were defined based on the absence of fluorescence in histological sections of control skin samples (Fig. A.1).

In the experiments here described, the most stable dye-loaded NPs were used, prepared according to the established protocols in our research group: the NB-loaded liposomes and ethosomes. Regarding PLA NPs, the results of NR-loaded PLA from Fernandes et al. were used for comparison in terms of HF penetration depth (Fernandes et al., 2015).

Drug non-encapsulation systems are commonly used in cosmetic products for the most diverse purposes. Based on a combination of suitable ingredients (Regulation EC No 1223/2009 of the European Parliament and of the Council of 30 November 2009 on cosmetic products, Official Journal of theEuropean Union; Buzek and Ask, 2009), these systems are easily prepared and approved for commercial use. Besides, they are in general stable as well as easily modifiable according to their purpose. In the scalp serum-like formulation, the amount of ethanol was adjusted according to the dye to be solubilized. However, the efficiency of such prepared systems is determined by their ability to deliver the cargo to the pretended target.

The permeation results of the NR when carried by the formulation are exemplified in Fig. 2. Besides the stratum corneum, fluorescence is detected along the entire follicular duct, the sebaceous glands, reaching the region of the bulb. When carried by this particular system, the hydrophobic NR is able to permeate deeply the HF and it localizes in the more lipidic environment of skin and HF. These results correlate well with the results obtained by Fernandes et al., when a drug encapsulation NP system was used to deliver the same hydrophobic molecule and the bulb region was also reached (Fernandes et al., 2015). In both studies, massage was not used (Lademann et al., 2011; Radtke et al., 2017). As known, skin and its appendage are more permeable to hydrophobic molecules then to hydrophilic ones. Therefore, one can expect that a formulation will succeed in the delivery of drugs that inherently already have a good permeation profile.

Based on the fact that the serum-like formulation and PLA NPs performed similarly in terms of follicular depth attained for this model molecule (NR), it can be said that the formulation is more advantageous. Its advantages are related to an easier preparation methodology that, when industrial production is desirable, has a huge impact on manufacturing costs.

Being more "water-friendly" than NR, more inherent difficulties could be foreseen in NB penetration along the follicular duct. On the other hand, in more hydrophobic media, NB has lower quantum yields than NR, being considerably more difficult to observe its fluorescence. In spite of these drawbacks, the penetration of NB can be observed along the HF till the region below the infundibulum (Fig. 3). Just a small amount of the dye seemed to be trapped in the stratum corneum and, as no fluorescence was detected in other skin layers, it does not reach the viable dermis in detectable amounts. The hair bulb does not show fluorescence (data not shown), meaning that with these nanoparticles,
A
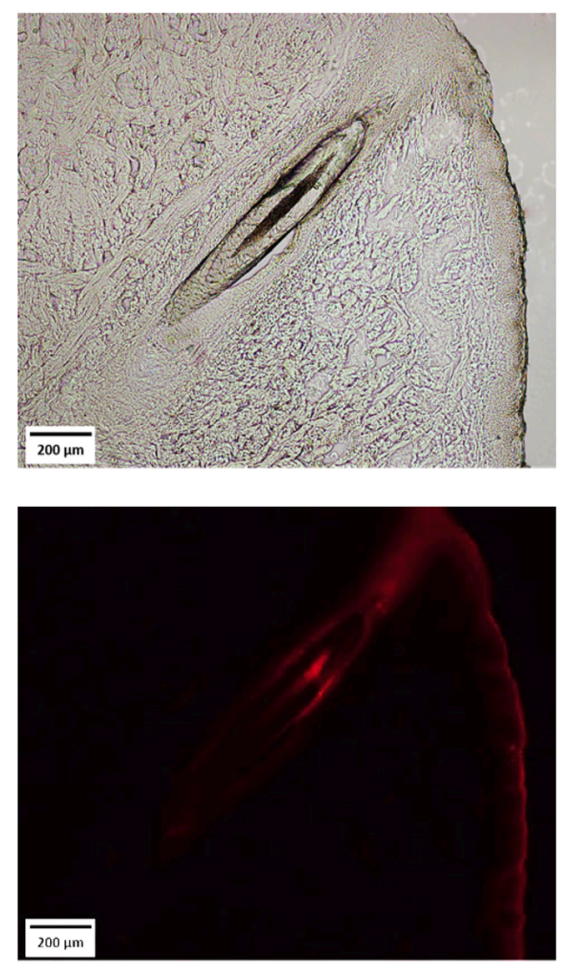

B

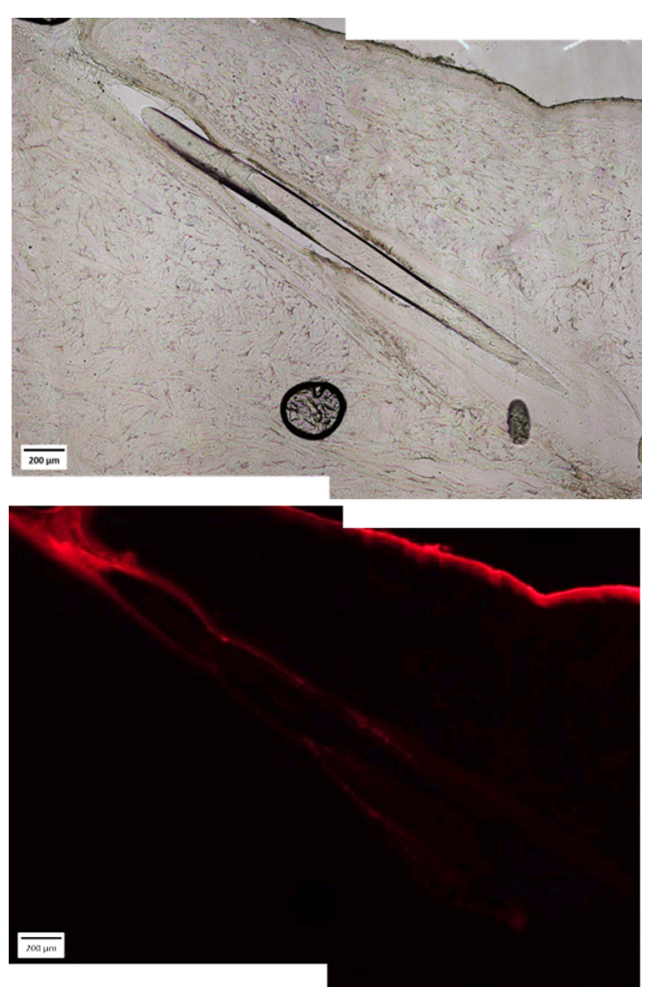

Fig. 3. Brightfield and fluorescence images of stratum corneum (A) and hair follicles (B) of cross-sectioned porcine skin, after $24 \mathrm{~h}$ of treatment with Nile Blue-loaded liposomes (Bar $=200 \mu \mathrm{m}, 800 \mathrm{~ms}$ of exposure). 

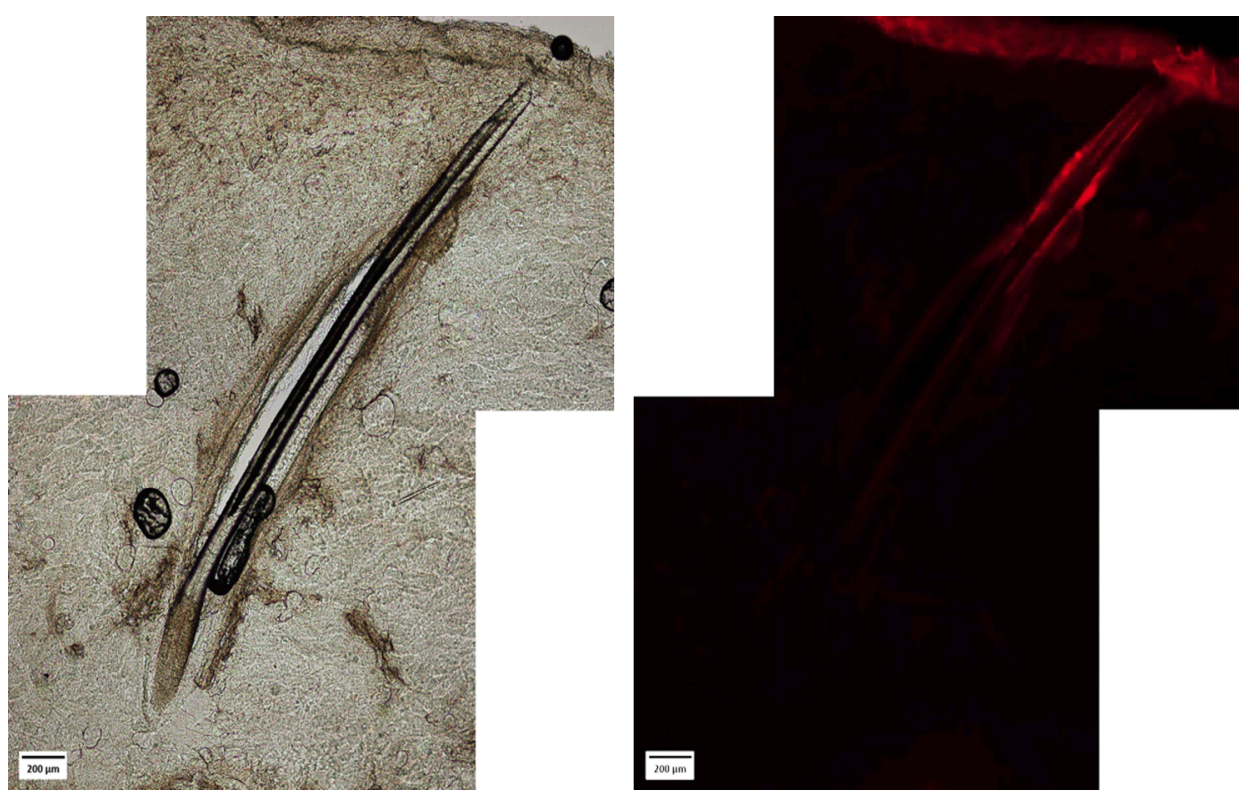

Fig. 4. Brightfield (left) and fluorescence (right) images of cross-sectioned porcine skin, after $24 \mathrm{~h}$ of treatment with Nile Blue-loaded ethosomes (Bar $=200 \mu \mathrm{m}$, 800 ms of exposure).

A
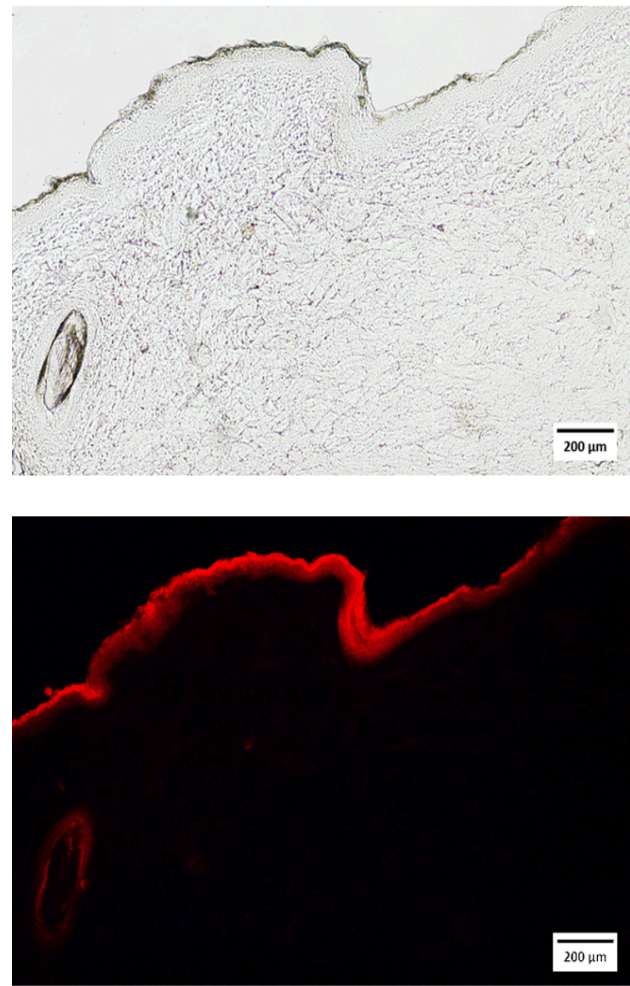

B
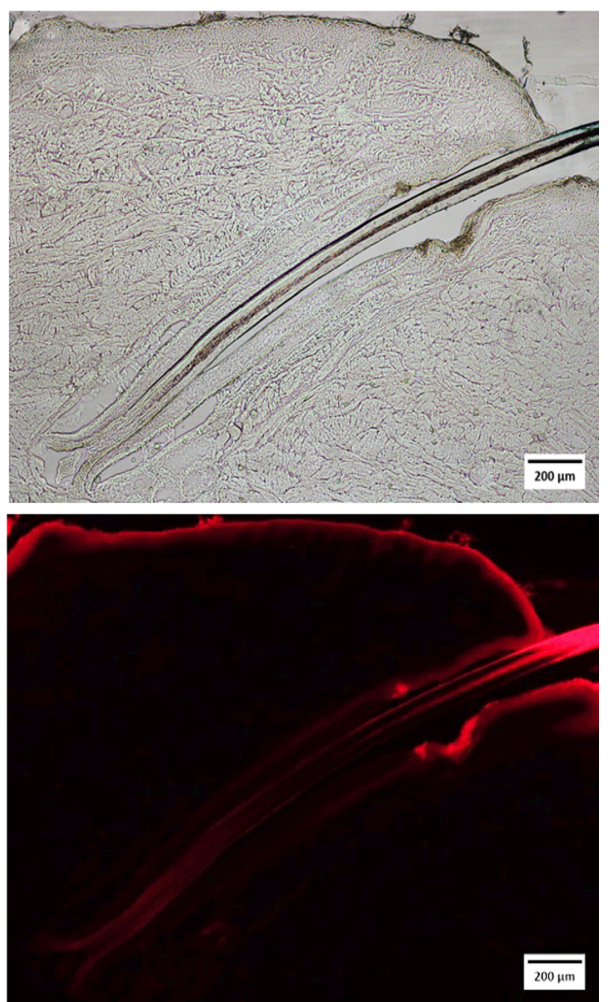

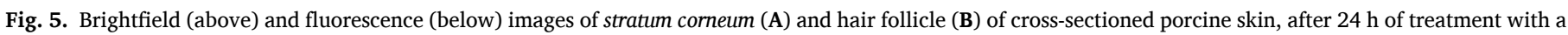
scalp serum formulation containing Nile Blue (Bar $=200 \mu \mathrm{m}, 800 \mathrm{~ms}$ of exposure).

NB did not reach the deepest region of the HF in detectable amounts. In fact, lipid-based particles are most studied for transdermal (Ashtikar et al., 2016; Ghanbarzadeh and Arami, 2013) or transfollicular applications (Jung et al., 2006; Serrano et al., 2015; Verma et al., 2016). To carry out their function as a transfollicular delivery system, it is enough that these particles reach the infundibulum region. This region is known as an area of additional absorption; in this follicle region there is an interruption, due to the continuous loss of epidermal differentiation, and it is supplied by a dense capillary network, which facilitate the transfollicular permeation of some substances (Blume-Peytavi and Vogt, 2011; Patzelt et al., 2011). Liposomes are also studied for the treatment of acne (Kumar and Banga, 2016), being sufficient that they reach the sebaceous glands. As these results are in line with the literature, liposomes will be more appropriate when targeting certain regions of the 
follicle not as deep as the bulb region.

Ethanol acts as a skin permeation enhancer, however, this effect heavily depends on the drug and vehicle in question (Carreras et al., 2020; Williams and Barry, 2012). According to Fig. 4, NB transported by ethosomes was found in follicular ducts of porcine skin after topical application, but it did not reach the bulb region. This permeation profile was similar to the one observed for NB when carried by liposomes.

Nanoparticles in general can be used for specific targeting of HFs (Fang et al., 2014; Lademann et al., 2008), since they can preferentially accumulate in the HFs while they cannot permeate the stratum corneum (Papakostas et al., 2011). Still, their tendency to accumulate in the follicular orifice may block the particles penetration into a deeper region of the follicular duct (Tran et al., 2017; Vogt et al., 2014). This occlusion effect could be present on the permeation results obtained for the lipid NPs (Figs. 3-4). As with liposomes, ethosomes are also extensively studied for transdermal applications (Monisha et al., 2019; Nainwal et al., 2019). One study evaluated ethosomes as a delivery vehicle for the pilosebaceous unit, however this formulation favors skin permeation and not its accumulation or transport along the follicle (Wilson et al., 2017).

Particle aggregation and penetration occlusion are not an issue regarding the serum-like formulation. In Fig. 5, a more uniform NB fluorescence is seen along the entire $\mathrm{HF}$, and specifically in the follicular bulb (Fig. 5B). Comparing to ethosomes or liposomes, the serum-like formulation delivers NB deeper than the tested NP systems after topical application in ex vivo porcine skin. Moreover, as noted for drug encapsulation systems, no fluorescence was detected in viable skin layers, the formulation was trapped in the stratum corneum which indicates HF selective delivery (Fig. 5A).

Looking at the qualitative results obtained with this comparative study, the serum-like formulation, developed for topical scalp application, performed better than the tested NP systems for NB, here used as a hydrophilic model molecule. In agreement with the particular aim of selectively target the hair bulb, as more independently of cargo hydrophilicity as possible, our choice lays on the formulation. However, a more complete picture must be obtained in future research by performing a quantitative approach to the amount of cargo that was indeed penetrating into the stratum corneum, hair follicles and viable skin, for each vehicle (Oliveira et al., 2020; Pereira et al., 2021; Tolentino et al., 2021).

Thought NPs are a hot topic, even within our research group, they must be selected as delivery system under specific requirements, e. g., when the target compound is unstable or when targeting a specific population of cells (Alsalhi et al., 2020; Jeong et al., 2019). However, in these situations, it will be necessary to carry out a whole set of optimizations depending on the particular molecule to be encapsulated. Serum-like formulations are less demanding in terms of optimization. Regarding this study and the model dyes used, the serum-like formulation was easily tuned for the delivery of both hydrophilic and hydrophobic molecules, in this case, to hair bulbs. However, other hydrophilic and lipophilic molecules should be also investigated, in order to better assess the "plasticity" and potential of this serum-like formulation as a HF delivery vehicle.

\section{Conclusion}

Drug delivery to the HFs has been under the research spotlight for the past two decades. The HFs offer new opportunities not only concerning HF associated diseases but also for specific therapies like immuno- and gene therapies, and for the development of new targeted cosmetics (Patzelt et al., 2008). In this work, our purpose was to reach a distinct follicle zone - the bulb. The hair bulb is a very important therapeutic and cosmetic target site of HFs. During the growth stage of the hair cycle, the bulb is the factory of the shaft, that under genetic control produce and give color to the hair. Hair bulb cells and their expressed genes and proteins are implicated on hair morphology determination and for that reason they are very appealing targets of innovative cosmetics. According to the work purpose, mapping the delivery to the bulb turn the histological evaluation unavoidable and essential. It was not so much the dye quantity that reaches the follicle that we were first interested in, but rather knowing whether it reaches the bulb or not. Hereafter, the obtained results are promising and leverage a quantitative evaluation of the cutaneous versus follicular pathways used by the different systems and their validation in human skin.

When the intention is to topically deliver substances to the HF, NPs are an obvious choice due to their intrinsic ability to penetrate and accumulate in HFs. This study proposes that scalp serum formulations must also be taken into consideration. Depending on the desired purpose, these systems have several advantages over particles: their preparation method is normally faster and easier; their composition can be easily modified and adjusted according to the molecule or molecules to be incorporated, and the scale up to industrial production is much faster with lower costs than for drug encapsulation nanosystems.

\section{CRediT authorship contribution statement}

Cristiana Costa: Methodology, Investigation, Data curation, Formal analysis, Software, Visualization, Writing - original draft. Bruno Fernandes: Methodology, Investigation, Data curation, Writing - review \& editing. Diana Guimarães: Methodology, Investigation. Eugénia Nogueira: Investigation. Madalena Martins: Investigation. Teresa Matamá: Conceptualization, Supervision, Writing - review \& editing. Artur Cavaco-Paulo: Supervision, Writing - review \& editing.

\section{Declaration of Competing Interest}

The authors declare that they have no known competing financial interests or personal relationships that could have appeared to influence the work reported in this paper.

\section{Acknowledgments}

The author Cristiana Costa would like to acknowledge her PhD scholarship funded by Portuguese Foundation for Science and Technology (FCT) (SFRH/BD/139522/2018). The author Bruno Fernandes would like to acknowledge his PhD scholarship funded by FCT (SFRH/ $\mathrm{BD} / 131824 / 2017)$. The author Diana Guimarães would like to acknowledge her PhD scholarship funded by FCT (SFRH/BD/140321/ 2018). This study was supported by the FCT under the scope of BioTecNorte operation (NORTE-01-0145-FEDER-000004) funded by the European Regional Development Fund under the scope of Norte2020 Programa Operacional Regional do Norte. The authors thank Seara S.A. and, in particular, Isabel Pinto (Quality Director, Seara SA) and her coworkers for providing and helping in the porcine skin sample collection.

\section{Appendix A. Supplementary data}

Supplementary data to this article can be found online at https://doi. org/10.1016/j.ijpharm.2021.120653.

\section{References}

Abd, E., Benson, H.E., Roberts, M., Grice, J., 2018. Follicular Penetration of Caffeine from Topically Applied Nanoemulsion Formulations Containing Penetration

Enhancers: In vitro Human Skin Studies. Skin Pharmacol. Physiol. 31 (5), 252-260. https://doi.org/10.1159/000489857.

Alsalhi, W., Alalola, A., Randolph, M., Gwillim, E., Tosti, A., 2020. Novel drug delivery approaches for the management of hair loss. Expert Opin. Drug Deliv. 17 (3), 287-295. https://doi.org/10.1080/17425247.2020.1723543.

Angelo, T., El-Sayed, N., Jurisic, M., Koenneke, A., Gelfuso, G.M., Cunha-Filho, M., Taveira, S.F., Lemor, R., Schneider, M., Gratieri, T., 2020. Effect of physical stimuli on hair follicle deposition of clobetasol-loaded Lipid Nanocarriers. Sci. Rep. 10 (1) https://doi.org/10.1038/s41598-019-56760-w. 
Ashtikar, M., Nagarsekar, K., Fahr, A., 2016. Transdermal delivery from liposomal formulations - Evolution of the technology over the last three decades. J. Control. Release 242, 126-140. https://doi.org/10.1016/j.jconrel.2016.09.008.

Badran, M., 2014. Formulation and in vitro evaluation of Flufenamic Acid Loaded deformable Liposomes for Improved Skin Delivery, Digest Journal of Nanomaterials and Biostructures.

Blume-Peytavi, U., Massoudy, L., Patzelt, A., Lademann, J., Dietz, E., Rasulev, U., Garcia Bartels, N., 2010. Follicular and percutaneous penetration pathways of topically applied minoxidil foam. Eur. J. Pharm. Biopharm. 76 (3), 450-453. https://doi.org/ 10.1016/j.ejpb.2010.06.010.

Blume-Peytavi, U., Vogt, A., 2011. Human hair follicle: reservoir function and selective targeting. Br. J. Dermatol. 165 (Suppl 2), 13-17. https://doi.org/10.1111/j.13652133.2011.10572.x

Bozzuto, G., Molinari, A., 2015. Liposomes as nanomedical devices. Int. J. Nanomedicine. https://doi.org/10.2147/IJN.S68861.

Buzek, J., Ask, B., 2009. Regulation (EC) No 1223/2009 of the European Parliament and of the Council of 30 November 2009 on cosmetic products. Off. J. Eur. Union L 342

Carreras, J.J., Tapia-Ramirez, W.E., Sala, A., Guillot, A.J., Garrigues, T.M., Melero, A., 2020. Ultraflexible lipid vesicles allow topical absorption of cyclosporin A. Drug Deliv. Transl. Res. 10, 486-497. https://doi.org/10.1007/s13346-019-00693-4.

Chen, M., Liu, X., Fahr, A., 2011. Skin penetration and deposition of carboxyfluorescein and temoporfin from different lipid vesicular systems: In vitro study with finite and infinite dosage application. Int. J. Pharm. 408, 223-234. https://doi.org/10.1016/j. ijpharm.2011.02.006

Czajkowska-Kośnik, A., Szekalska, M., Winnicka, K., 2019. Nanostructured lipid carriers: A potential use for skin drug delivery systems. Pharmacol. Reports. https://doi.org/ 10.1016/j.pharep.2018.10.008

Danaei, M., Dehghankhold, M., Ataei, S., Hasanzadeh Davarani, F., Javanmard, R., Dokhani, A., Khorasani, S., Mozafari, M.R., 2018. Impact of particle size and polydispersity index on the clinical applications of lipidic nanocarrier systems. Pharmaceutics. https://doi.org/10.3390/pharmaceutics10020057.

Fang, C.-L., Aljuffali, I.A., Li, Y.-C., Fang, J.-Y., 2014. Delivery and targeting of nanoparticles into hair follicles. Ther. Deliv. 5, 991-1006. https://doi.org/10.4155/ tde.14.61.

Fernandes, B., Silva, R., Ribeiro, A., Matamá, T., Gomes, A.C., Cavaco-Paulo, A.M., 2015 Improved Poly (D, L-lactide) nanoparticles-based formulation for hair follicle targeting. Int. J. Cosmet. Sci. 37, 282-290. https://doi.org/10.1111/ics.12197.

Ghanbarzadeh, S., Arami, S., 2013. Enhanced transdermal delivery of diclofenac sodium via conventional liposomes, ethosomes, and transfersomes. Biomed Res. Int. 2013 https://doi.org/10.1155/2013/616810.

Guimarães, D., Noro, J., Loureiro, A., Lager, F., Renault, G., Cavaco-Paulo, A., Nogueira, E., 2020. Increased Encapsulation Efficiency of Methotrexate in Liposomes for Rheumatoid Arthritis Therapy. Biomedicines 8, 630. https://doi.org/10.3390/ biomedicines8120630.

Hammond, S.A., Tsonis, C., Sellins, K., Rushlow, K., Scharton-Kersten, T., Colditz, I., Glenn, G.M., 2000. Transcutaneous immunization of domestic animals: Opportunities and challenges. Adv. Drug Deliv. Rev. 43, 45-55. https://doi.org/ 10.1016/S0169-409X(00)00076-4.

Jeong, W.Y., Kim, S., Lee, S.Y., Lee, H., Han, D.W., Yang, S.Y., Kim, K.S., 2019. Transdermal delivery of Minoxidil using HA-PLGA nanoparticles for the treatment in alopecia. Biomater. Res. 23 https://doi.org/10.1186/s40824-019-0164-z.

Jung, S., Otberg, N., Thiede, G., Richter, H., Sterry, W., Panzner, S., Lademann, J., 2006 Innovative liposomes as a transfollicular drug delivery system: Penetration into porcine hair follicles. J. Invest. Dermatol. 126, 1728-1732. https://doi.org/ 10.1038/sj.jid.5700323.

Knorr, F., Lademann, J., Patzelt, A., Sterry, W., Blume-Peytavi, U., Vogt, A., 2009. Follicular transport route - Research progress and future perspectives. Eur. J. Pharm. Biopharm. 71, 173-180. https://doi.org/10.1016/j.ejpb.2008.11.001.

Kumar, V., Banga, A.K., 2016. Intradermal and follicular delivery of adapalene liposomes. Drug Dev. Ind. Pharm. 42, 871-879. https://doi.org/10.3109/ 03639045.2015.1082580.

Lademann, J., Knorr, F., Richter, H., Blume-Peytavi, U., Vogt, A., Antoniou, C. Sterry, W., Patzelt, A., 2008. Hair follicles - An efficient storage and penetration pathway for topically applied substances: Summary of recent results obtained at the Center of Experimental and Applied Cutaneous Physiology. Charité Universitätsmedizin Berlin, Germany, in: Skin Pharmacology and Physiology. 150-155. https://doi.org/10.1159/000131079.

Lademann, J., Richter, H., Schaefer, U.F., Blume-Peytavi, U., Teichmann, A., Otberg, N., Sterry, W., 2006. Hair follicles - a long-term reservoir for drug delivery. Skin Pharmacol. Physiol. 19, 232-236. https://doi.org/10.1159/000093119.

Lademann, J., Richter, H., Schanzer, S., Knorr, F., Meinke, M., Sterry, W., Patzelt, A., 2011. Penetration and storage of particles in human skin: Perspectives and safety aspects. Eur. J. Pharm. Biopharm. https://doi.org/10.1016/j.ejpb.2010.10.015.

Lauterbach, A., Müller-Goymann, C.C., 2015. Applications and limitations of lipid nanoparticles in dermal and transdermal drug delivery via the follicular route. Eur. J. Pharm. Biopharm. https://doi.org/10.1016/j.ejpb.2015.06.020.

Martinez, V., Henary, M., 2016. Nile Red and Nile Blue: Applications and Syntheses of Structural Analogues. Chem. - A Eur. J. 22, 13764-13782. https://doi.org/10.1002 chem. 201601570

Mittal, A., Raber, A.S., Schaefer, U.F., Weissmann, S., Ebensen, T., Schulze, K., Guzmán, C.A., Lehr, C.M., Hansen, S., 2013. Non-invasive delivery of nanoparticles to hair follicles: A perspective for transcutaneous immunization. Vaccine 31, 3442-3451. https://doi.org/10.1016/j.vaccine.2012.12.048.

Monisha, C., Ganesh, G., Mythili, L., Radhakrishnan, K., 2019. A Review on Ethosomes for Transdermal Application. Res. J. Pharm. Technol. 12, 3133. https://doi.org/ 10.5958/0974-360x.2019.00529.8.
Morganti, P., 2010. Use and potential of nanotechnology in cosmetic dermatology. Clin. Cosmet. Investig. Dermatol. https://doi.org/10.2147/ccid.s4506.

Nainwal, N., Jawla, S., Singh, R., Saharan, V.A., 2019. Transdermal applications of ethosomes-a detailed review. J. Liposome Res. https://doi.org/10.1080/ 08982104.2018.1517160.

Oliveira, A.C.S., Oliveira, P.M., Cunha-Filho, M., Gratieri, T., Gelfuso, G.M., 2020. Latanoprost Loaded in Polymeric Nanocapsules for Effective Topical Treatment of Alopecia. AAPS PharmSciTech 21, 1-7. https://doi.org/10.1208/s12249-02001863-1.

Ossadnik, M., Richter, H., Teichmann, A., Koch, S., Schäfer, U., Wepf, R., Sterry, W., Lademann, J., 2006. Investigation of differences in follicular penetration of particleand nonparticle-containing emulsions by laser scanning microscopy. Laser Phys. 16, 747-750. https://doi.org/10.1134/s1054660x06050033.

Papakostas, D., Rancan, F., Sterry, W., Blume-Peytavi, U., Vogt, A., 2011. Nanoparticles in dermatology. Arch. Dermatol. Res. 303, 533-550. https://doi.org/10.1007/ s00403-011-1163-7.

Patzelt, A., Knorr, F., Blume-Peytavi, U., Sterry, W., Lademann, J., 2008. Hair follicles, their disorders and their opportunities. Drug Discov. Today Dis. Mech. 5, e173-e181. https://doi.org/10.1016/j.ddmec.2008.04.006.

Patzelt, A., Lademann, J., 2020. Recent advances in follicular drug delivery of nanoparticles. Expert Opin. Drug Deliv. 17, 49-60. https://doi.org/10.1080/ 17425247.2020.1700226.

Patzelt, A., Richter, H., Knorr, F., Schäfer, U., Lehr, C.M., Dähne, L., Sterry, W., Lademann, J., 2011. Selective follicular targeting by modification of the particle sizes. J. Control. Release. https://doi.org/10.1016/j.jconrel.2010.11.015.

Pereira, M.N., Tolentino, S., Pires, F.Q., Anjos, J.L.V., Alonso, A., Gratieri, T., CunhaFilho, M., Gelfuso, G.M., 2021. Nanostructured lipid carriers for hair follicle-targeted delivery of clindamycin and rifampicin to hidradenitis suppurativa treatment. Colloids Surfaces B Biointerfaces 197, 111448. https://doi.org/10.1016/j. colsurfb.2020.111448.

Pilch, E., Musiał, W., 2018. Liposomes with an ethanol fraction as an application for drug delivery. Int. J. Mol. Sci. https://doi.org/10.3390/ijms19123806.

Putri, D.C.A., Dwiastuti, R., Marchaban, M., Nugroho, A.K., 2017. Optimization of mixing temperature and sonication duration in liposome preparation. J. Pharm. Sci. Community 14, 79-85. https://doi.org/10.24071/jpsc.142728.

Radtke, M., Patzelt, A., Knorr, F., Lademann, J., Netz, R.R., 2017. Ratchet effect for nanoparticle transport in hair follicles. Eur. J. Pharm. Biopharm. 116, 125-130. https://doi.org/10.1016/j.ejpb.2016.10.005.

Rancan, F., Papakostas, D., Hadam, S., Hackbarth, S., Delair, T., Primard, C., Verrier, B., Sterry, W., Blume-Peytavi, U., Vogt, A., 2009. Investigation of polylactic acid (PLA) nanoparticles as drug delivery systems for local dermatotherapy. Pharm. Res. 26, 2027-2036. https://doi.org/10.1007/s11095-009-9919-x.

Regulation (EC) No 1223/2009 of the European Parliament and of the Council of 30 November 2009 on cosmetic products, Official Journal of the European Union, 2009. 01/05/2020 (Accessed 14 September 2020).

Rosen, J., Landriscina, A., Friedman, A., 2015. Nanotechnology-Based Cosmetics for Hair Care. Cosmetics 2, 211-224. https://doi.org/10.3390/cosmetics2030211.

Sala, M., Diab, R., Elaissari, A., Fessi, H., 2018. Lipid nanocarriers as skin drug delivery systems: Properties, mechanisms of skin interactions and medical applications. Int. J. Pharm. https://doi.org/10.1016/j.ijpharm.2017.10.046.

Santos, G.A., Angelo, T., Andrade, L.M., Silva, S.M.M., Magalhães, P.O., Cunha-Filho, M., Gelfuso, G.M., Taveira, S.F., Gratieri, T., 2018. The role of formulation and follicular pathway in voriconazole cutaneous delivery from liposomes and nanostructured lipid carriers. Colloids Surfaces B Biointerfaces 170, 341-346. https://doi.org/ 10.1016/j.colsurfb.2018.06.037.

Schafer-Korting, M., Mehnert, W., Korting, H.-C., 2007. Lipid nanoparticles for improved topical application of drugs for skin diseases々. Adv. Drug Deliv. Rev. 59, 427-443. https://doi.org/10.1016/j.addr.2007.04.006.

Serrano, G., Almudéver, P., Serrano, J.M., Cortijo, J., Faus, C., Reyes, M., Expósito, I., Torrens, A., Millán, F., 2015. Microneedling dilates the follicular infundibulum and increases transfollicular absorption of liposomal sepia melanin. Clin. Cosmet. Investig. Dermatol. 8, 313-318. https://doi.org/10.2147/CCID.S77228.

Simon, G.A., Maibach, H.I., 2000. The pig as an experimental animal model of percutaneous permeation in man: Qualitative and quantitative observations - An overview. Skin Pharmacol. Appl. Skin Physiol. https://doi.org/10.1159/000029928.

Teichmann, A., Heuschkel, S., Jacobi, U., Presse, G., Neubert, R.H.H., Sterry, W., Lademann, J., 2007. Comparison of stratum corneum penetration and localization of a lipophilic model drug applied in an o/w microemulsion and an amphiphilic cream. Eur. J. Pharm. Biopharm. 67, 699-706. https://doi.org/10.1016/j. ejpb.2007.04.006.

Tolentino, S., Pereira, M.N., Cunha-Filho, M., Gratieri, T., Gelfuso, G.M., 2021. Targeted clindamycin delivery to pilosebaceous units by chitosan or hyaluronic acid nanoparticles for improved topical treatment of acne vulgaris. Carbohydr. Polym. 253, 117295 https://doi.org/10.1016/j.carbpol.2020.117295.

Tran, N.B.N.N., Knorr, F., Mak, W.C., Cheung, K.Y., Richter, H., Meinke, M., Lademann, J., Patzelt, A., 2017. Gradient-dependent release of the model drug TRITC-dextran from FITC-labeled BSA hydrogel nanocarriers in the hair follicles of porcine ear skin. Eur. J. Pharm. Biopharm. 116, 12-16. https://doi.org/10.1016/j. ejpb.2016.09.016.

Ushirobira, C.Y., Afiune, L.A.F., Pereira, M.N., Cunha-Filho, M., Gelfuso, G.M., Gratieri, T., 2020. Dutasteride nanocapsules for hair follicle targeting: Effect of chitosan-coating and physical stimulus. Int. J. Biol. Macromol. 151, 56-61. https:// doi.org/10.1016/j.ijbiomac.2020.02.143.

Verma, A., Jain, S.K., Jain, A., Hurkat, P., 2016. Transfollicular drug delivery: current perspectives. Res. Reports Transdermal Drug Deliv. 5, 1. https://doi.org/10.2147/ rrtd.s75809. 
Vogt, A., Combadiere, B., Hadam, S., Stieler, K.M., Lademann, J., Schaefer, H., Autran, B., Sterry, W., Blume-Peytavi, U., 2006. $40 \mathrm{~nm}$, but not 750 or 1,500 nm, nanoparticles enter epidermal CD1a+ cells after transcutaneous application on human skin. J. Invest. Dermatol. 126, 1316-1322. https://doi.org/10.1038/sj. jid.5700226.

Vogt, A., Mandt, N., Lademann, J., Schaefer, H., Blume-Peytavi, U., 2005. Follicular targeting-a promising tool in selective dermatotherapy. J. Investig. Dermatol. Symp. Proc. 10, 252-255. https://doi.org/10.1111/j.1087-0024.2005.10124.x.

Vogt, A., Rancan, F., Ahlberg, S., Nazemi, B., Choe, C.S., Darvin, M.E., Hadam, S., BlumePeytavi, U., Loza, K., Diendorf, J., Epple, M., Graf, C., Ruhl, E., Meinke, M.C., Lademann, J., 2014. Interaction of dermatologically relevant nanoparticles with skin cells and skin. Beilstein J. Nanotechnol. 5, 2363-2373. https://doi.org/10.3762/ binano.5.245.

Williams, A.C., Barry, B.W., 2012. Penetration enhancers. Adv. Drug Deliv. Rev. https:// doi.org/10.1016/j.addr.2012.09.032.

Wilson, V., Siram, K., Rajendran, S., Sankar, V., 2017. Development and evaluation of finasteride loaded ethosomes for targeting to the pilosebaceous unit. Artif. Cells, Nanomedicine, Biotechnol. 46, 1-10. https://doi.org/10.1080/ 21691401.2017.1396221.

Wosicka, H., Cal, K., 2010. Targeting to the hair follicles: current status and potential. J. Dermatol. Sci. 57, 83-89. https://doi.org/10.1016/j.jdermsci.2009.12.005. 